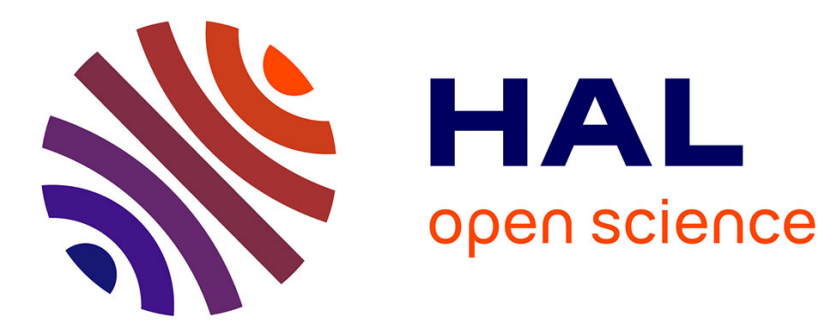

\title{
Les nouvelles tours de Londres comme marqueurs des mutations d'une métropole globale
}

\author{
Manuel Appert
}

\section{To cite this version:}

Manuel Appert. Les nouvelles tours de Londres comme marqueurs des mutations d'une métropole globale. Observatoire de la société britannique, 2011, 11, pp.105-122. 10.4000/osb.1243 . halshs00841529

\section{HAL Id: halshs-00841529 \\ https://shs.hal.science/halshs-00841529}

Submitted on 5 Jul 2013

HAL is a multi-disciplinary open access archive for the deposit and dissemination of scientific research documents, whether they are published or not. The documents may come from teaching and research institutions in France or abroad, or from public or private research centers.
L'archive ouverte pluridisciplinaire HAL, est destinée au dépôt et à la diffusion de documents scientifiques de niveau recherche, publiés ou non, émanant des établissements d'enseignement et de recherche français ou étrangers, des laboratoires publics ou privés. 


\section{Les nouvelles tours de Londres comme marqueurs des mutations d'une métropole globale}

\section{Working paper}

\section{Manuel Appert}

Manuel Appert est docteur en géographie et aménagement, maître de conférences à l'université Lyon2 (Université de Lyon) et membre du laboratoire Environnement, Ville, Société (EVS - UMR 5600) depuis 2006. Le fil conducteur de ses recherches est la métropolisation, d'abord abordée sous l'angle des interactions transports - occupation du sol, puis orientée depuis 2007 vers les enjeux de gouvernance des formes urbaines et plus particulièrement celle des tours.

\section{Résumé}

Depuis 2001, plus d'une centaine de projets de tours a été proposée à Londres. Dans le contexte du développement durable et de la globalisation, elles sont une solution privilégiée pour la densification urbaine mais aussi des marqueurs paysagers pour les acteurs qui les instrumentalisent. Toutefois, elles suscitent aussi dans leur sillage des controverses qu'une législation développée à l'échelle de la Greater London Authority peine à apaiser. Des espaces de la métropole semblent plus concernés par ces conflits que d'autres, laissant apparaître des contraintes et opportunités qui sont à expliquer. Une double différenciation spatiale se dessine associée aux caractéristiques des projets et à la variabilité des contextes locaux et métropolitains. Une géographie des tours londoniennes permettra, en amont, de comprendre leurs localisation, fonctions et représentations en fonction des mutations socio-économiques, culturelles et politiques de cette métropole hétérogène et globale.

Mots clés : gratte-ciel, urbanisme, régulation, localisation, fonctions économiques, ville durable, paysage urbain, skyline, Londres

\section{Introduction}

La tour est devenue l'un des marqueurs les plus frappants de la métropolisation, y compris dans le bastion du victorianisme qu'était la City de Londres. Le début du dernier film des Monthy Python, The Meaning of Life (1983) qui voit l'assaut d'un gratte-ciel de Manhattan par le vénérable immeuble victorien d'une poussiéreuse firme financière ne serait plus possible aujourd'hui : l'essor de la globalisation économique, du marketing urbain et l'interprétation des préceptes du développement durable ont transformé la donne. En effet, plus d'une cinquantaine de tours sont en passe de transformer radicalement le skyline de Londres, modifiant cette ligne d'horizon chargée $d$ 'histoire et de symboles. Initialement conçues comme infrastructure du système de production de l'économie tertiaire, les tours sont aujourd'hui devenues des investissements dans le marché immobilier financiarisé des métropoles. Elles sont aussi une solution résidentielle en réponse aux exigences de densification et de régénération urbaine pour un développement urbain durable. Par leur proéminence et leur dessin, elles sont par ailleurs des marqueurs paysagers distinctifs non seulement pour les entreprises, mais aussi pour les résidents et visiteurs. Elles sont enfin de véri- 
tables leviers de communication pour les acteurs qui font le choix de leur instrumentalisation dans une perspective de marketing urbain. Pour toutes ces raisons, elles suscitent des controverses dans le processus d'urbanisme et au-delà, dans la presse locale et nationale. Une règlementation visant à réguler les conflits a d'ailleurs été mise en place par la Greater London Authority en 2007 sans toutefois apaiser les tensions entre les acteurs de la ville.

Des espaces de la métropole semblent plus concernés par ces conflits que d'autres, laissant apparaître des contraintes et opportunités qui sont à expliquer. Une double différenciation spatiale se dessine. Elle est liée d'une part aux caractéristiques des projets et d'autre part, à la variabilité des contextes locaux et des dynamiques métropolitaines. Une géographie des tours londoniennes permettrait ainsi, en amont, de comprendre leurs localisation, fonctions et représentations en fonction des mutations socio-économiques, culturelles et politiques de cette métropole hétérogène et globale.

\section{Tours et métropolisation}

Retracer le retour des tours à Londres nécessite, dans un premier temps, une mise en perspective du cas londonien dans le processus de verticalisation des villes mondiales.

Le développement rapide des tours dans le monde s'explique d'abord par l'urbanisation que connaissent les pays dont la transition démographique est achevée ou en cours d'achèvement. Dans ce contexte, la croissance urbaine rapide des villes des pays émergents tels que la Chine ou l'Inde se traduit souvent par une verticalisation des complexes résidentiels via l'utilisation de solutions urbanistiques standardisées qui reposent, comme dans les années 1960-1970 en Europe, sur de I'habitat collectif (figure 1). Si l'on considère les seules villes mondiales, les tours sont davantage une réponse au système de production de l'économie contemporaine. Au même titre que les plates-formes aéroportuaires ou les réseaux de télécommunication, la tour de bureaux est un hub infrastructurel qui permet aux métropoles d'encadrer l'économie mondialisée ${ }^{1}$.

Elles permettent ainsi d'abriter les cols blancs ainsi que leurs réseaux techniques tout comme elles favorisent la concentration et les relations de face à face, nécessaires au fonctionnement de l'économie métropolitaine. Devenues biens marchands, elles participent aussi à la constitution et à la dynamique des marchés immobiliers urbains en devenant cible d'investissements. Enfin, par leur proéminence et leur apparence, elles sont un signe distinctif pour les entreprises qui les construisent et qui les occupent ${ }^{2}$. Leurs caractéristiques ainsi que leur position dans le skyline confèrent à leurs promoteurs ou leurs occupants, une visibilité et un prestige qui est à associer à la représentation collective de « la réussite économique et [de] la puissance politique des États-Unis au cours du $20^{\mathrm{e}}$ siècle $^{3}$ ".

\footnotetext{
${ }^{1}$ Graham S., Marvin S., Splintering Urbanism: Networked Infrastructures, Technological Mobilites and the Urban Condition, Routledge, 2001.

${ }^{2}$ D. McNeill, Skyscraper geography, in Progress in Human Geography, vol. 29, n¹, 2005, p. 41-55.

3 J. Monnet, La symbolique des lieux : pour une géographie des relations entre espace, pouvoir et identité, in Cybergeo, 2007, http://www.cybergeo.eu/index5316.html (consulté le 16 janvier 2011).
} 
Figure 1 - Tours de plus de 150m construites dans le Monde de 1920 à 2009

M. Appert, 2010, d'après les données du Council on Tall Buildings and Urban Habitat

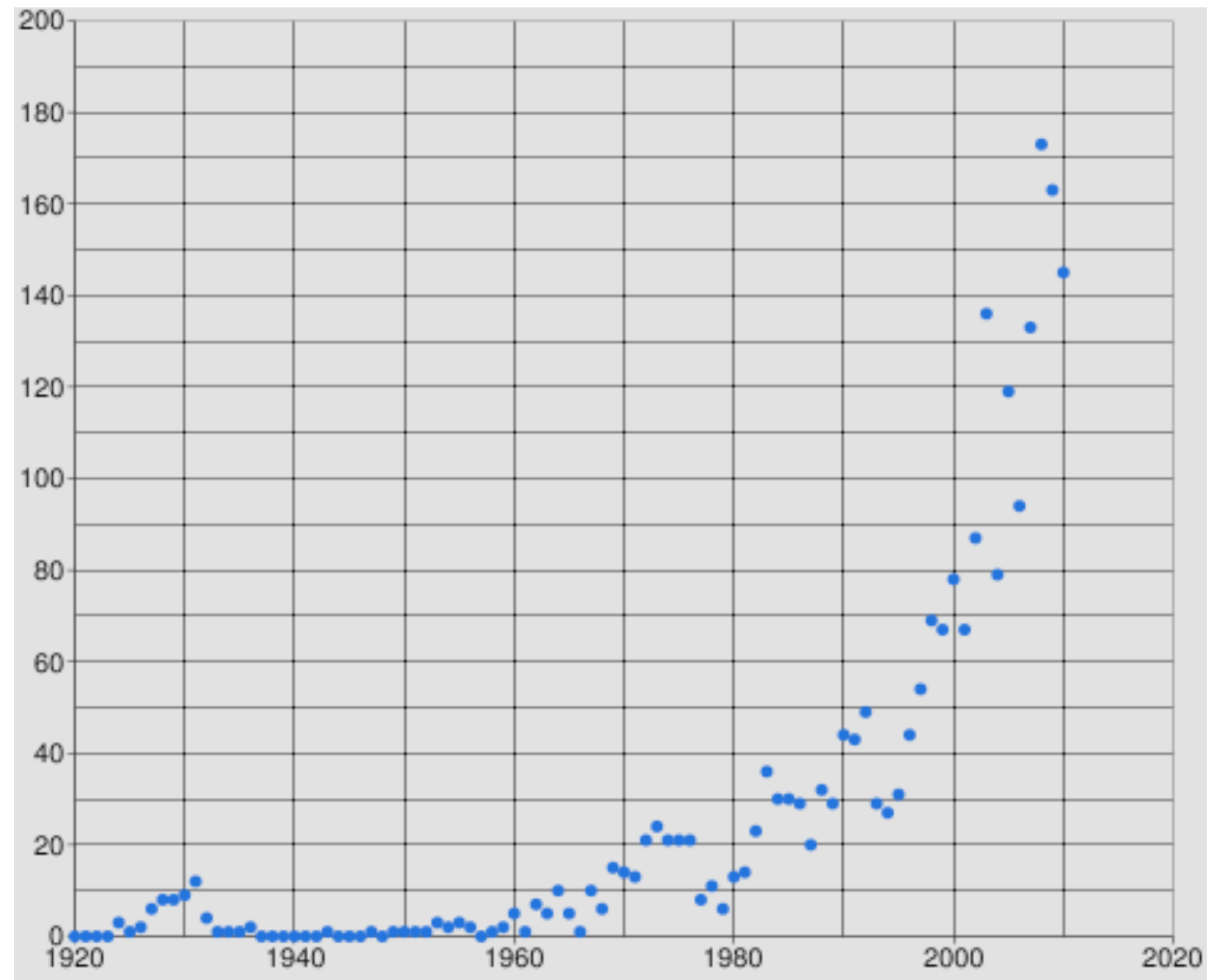

Cependant, la pertinence des tours ne relève pas seulement de la sphère économique ; elle est, dans le contexte du développement durable, un enjeu environnemental. La question recouvre trois dimensions : les conditions de construction des tours, la performance énergétique de leur architecture et leur localisation. En ce qui concerne la dernière dimension, si densité et tours ne sont pas fortement corrélées, elles tendent en revanche à maximiser les bienfaits de la coordination des transports et de l'usage du sol dans des sites à forte accessibilité ${ }^{4}$.

Outre l'importance fonctionnelle ou l'efficacité énergétique supposées des tours, leur architecture peut être un levier de marketing urbain en modifiant le paysage urbain et en mobilisant des discours performatifs. Aujourd'hui, c'est surtout la signature du "starchitecte » qui prime", autrement dit, la marque de fabrique d'un cabinet, déclinée selon les contextes de réalisation. Le Gherkin à Londres du cabinet Foster and Partners ou bien encore la Torre Agbar de Jean Nouvel à Barcelone comptent parmi les exemples les plus emblématiques.

${ }^{4}$ Urban Task Force, Toward an urban Renaissance, 1999.

M. Appert, Métropolisation, mobilités quotidiennes et forme urbaine : le cas de Londres, in Géocarrefour, vol. 79, $\mathrm{n}^{\circ} 2,2004$, p. 109-118.

${ }^{5}$ D. McNeill, Skyscraper geography, in Progress in Human Geography, vol. 29, n¹, 2005, p. 41-55. 
Le gratte-ciel est enfin conçu comme un élément clé de l'exercice de composition paysagère qui dépasse le seul affichage d'une ou plusieurs identité(s) privée(s). L'analyse du discours des architectes met en évidence un paramètre fondamental : les tours permettent de sculpter le skyline. Pour C. Pelli ${ }^{6}$, les vrais gratte-ciel sont ces édifices « chargés de responsabilités en termes de représentation, qui, par leur hauteur, agissent comme des marqueurs des lieux, des sculpteurs de silhouette urbaine et médias d'image collective ${ }^{7}$ ". Le gratte-ciel mimerait ainsi à des fins commerciales le rôle des édifices religieux et civiques qui ont longtemps, seuls, marqué de leur présence les villes européennes. Cela tend à expliquer la vivacité de l'opposition aux tours dans ces même villes où la charge symbolique des édifices historiques participe de la mémoire et, par son truchement, de l'identité collective.

Le cas de Londres révèle, comme pour d'autres villes européennes, une grande timidité voire un embarra vis à vis des tours. L'histoire des quelques dizaines d'édifices de grande hauteur dans la ville n'est pas linéaire et révèle des périodes d'accélération et de stabilisation. Elles sont à associer à l'évolution de la législation, aux dynamiques démographiques, à la conjoncture économique mais aussi à l'importance de quelques personnalités qui ont joué un rôle déterminant dans la multiplication des projets durant les années 2000.

\section{Le retour des tours à Londres et l'évolution de leur régulation}

Depuis les collines environnantes de Londres telle que Parliament Hill, la ville déploie une masse urbaine horizontale que quelques édifices proéminents viennent ponctuer. La ligne d'horizon ou skyline qui en résulte rappelle davantage Paris et Vienne que New York et Chicago (figure 2). Jusque dans les années 1960, seules les Houses of Parliament (101m et $86 \mathrm{~m})$, St Paul's (111m), plusieurs autres églises ainsi que les cheminées héritées de l'ère industrielle se dressaient audessus d'une canopée urbaine relativement homogène et basse.

Figure 2 - Centre de Londres depuis Parliament Hill en 2011

M. Appert, 2011

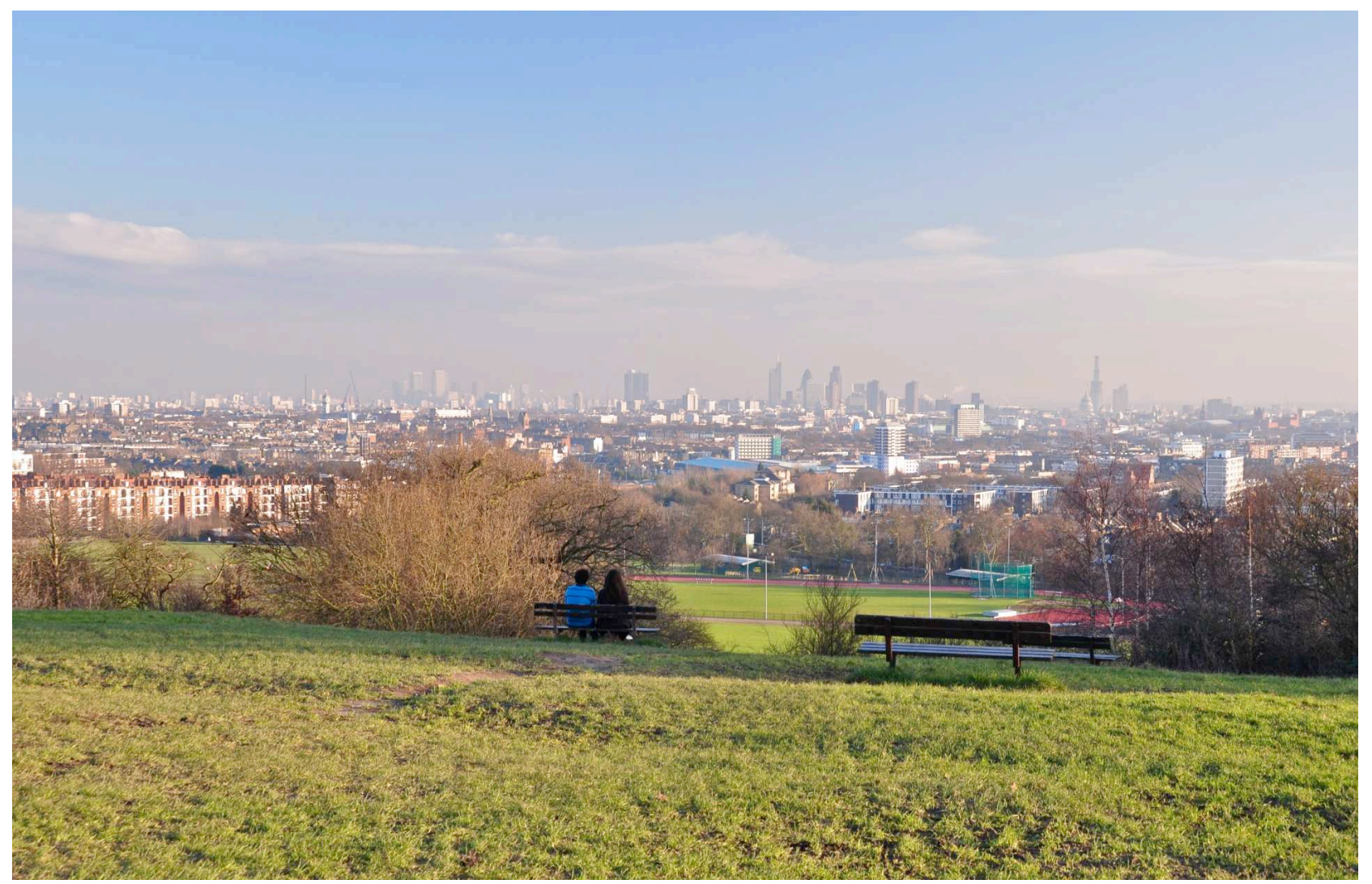


Au cours de la longue histoire de Londres, ses édifices religieux ont en effet longtemps trôné sans partage jusqu'à ce que les pouvoirs politique puis industriel ne viennent leur disputer leur domination. A partir des années 1960 et jusqu'à la fin des années 1970, le modernisme architectural alors en vogue dans les villes des pays industrialisés s'est traduit par une multiplication des tours de bureaux (Millbank Tower, 1963, 118m ; Centre Point, 1966, 117m), de télécommunication (BT Tower, 1965, 188m), de services publics (Guy's Hospital Tower, 1974, 143m) et de logements, connues (Balfron Tower, 1967, 84m ; Trellick Tower, 1972, 98m), ou moins connues. Parmi ces dernières, des réalisations aux qualités matérielles médiocres ont provoqué le mécontentement et la résistance du public et des élus du Greater London Council. Réticences qui se sont ensuite transformées progressivement en rejet lorsque la tour de logements sociaux de Ronan Point à Newham s'écroula en 1968. Conjugué aux effets de la crise économique dès 1974, un très net ralentissement de la construction de tours dans la ville se produisit (figure 3). Au cours de la deuxième partie du XXème siècle, l'évolution du nombre de projets de tours a donc fluctué selon la conjoncture économique, l'adhésion puis le rejet des préceptes du modernisme et de la Charte d'Athènes ${ }^{8}$, la montée des valeurs patrimoniales, mais aussi selon l'évolution de la législation. Londres n'a jamais embrassé la verticalité et s'est toujours attachée, de façon plus ou moins formalisée et avec plus ou moins de succès, à contrôler sa canopée urbaine. Depuis 1894, c'est un souci de préservation des vues de monuments stratégiques dont la cathédrale Saint Paul qui préside à l'orientation de la législation. Plus que sur le nombre de tours, la législation joue un rôle déterminant dans la localisation des projets depuis les années 1990.

Le London Building Act de $1894^{\circ}$, dont l'objet était d'assurer un minimum de lumière pour chaque logement, resta le seul outil de régulation de hauteur des nouvelles constructions jusqu'au lendemain de la seconde guerre mondiale, période à partir de laquelle des mutations technologiques et sociales ainsi que le besoin de reconstruction des sites bombardés allaient créer un nouveau climat $^{10}$. En 1947, la City de Londres intégra en effet dans sa législation une règlementation de l'usage du sol par coefficient d'occupation des sites. Concernant prioritairement les immeubles de bureaux, cette législation limitait la densité et indirectement la hauteur des nouvelles constructions en cours et à venir ${ }^{11}$.

Deux motivations principales avaient conduit à ces restrictions; il s'agissait d'une part, comme pour le London Building Act de 1894, d'assurer un minimum d'air pur et de lumière aux édifices existants et d'autre part d'assurer la prééminence de la cathédrale Saint Paul dans le skyline de la City. Bien que la rigueur en ait été réduite en 1956 pour permettre une reconstruction plus intensive de la City de Londres, cette législation a créé un précédent pour la protection des vues de $S t$ Paul's, monument devenu emblématique de la résistance des Londoniens face aux bombes de la Luftwaffe. Les directives générales qui se sont ensuite multipliées à l'échelle du London County Council dans les années 1960 se voulaient pragmatiques en permettant d'évaluer les nouvelles constructions au cas par cas, en fonction de leur qualité ${ }^{12}$. La multiplication des projets de tours au

\footnotetext{
${ }^{8}$ La Charte d'Athènes représente l'aboutissement du Congrès international d'architecture moderne sous l'égide de Le Corbusier en 1933. Composée de 95 points, elle pose les principes d'une extension rationnelle des villes par l'usage des tours et la séparation des fonctions et des circulations.

${ }^{9}$ R. Dennis, 'Babylonian Flats' in Victorian and Edwardian London, in The London Journal , vol. 33, n³, 2008, p. 233247.

${ }^{10}$ T. Catchpole, London Skylines: A study of High Buildings and Views, London Research Centre, vol. 33, 1987.

${ }^{11}$ R.D. Simon, Skyscrapers and the new London skyline : 1945-1991, The Electronic Journal of Architecture, vol.5, $\mathrm{n}^{\circ} 2$, 1996.

${ }^{12} \mathrm{M}$. Appert, Ville globale versus ville patrimoniale ? Des tensions entre libéralisation de la skyline de Londres et préservation des monuments et vues historiques. Revue Géographique de l’Est, vol. 48, n¹-2, 2008, http://rge.revues.org/index1154.htm, (consulté le 12 janvier 2011).
} 
début des années 1960 constituait de fait un dilemme pour les urbanistes. En 1968, 109 bâtiments de plus de $46 \mathrm{~m}$ furent érigés et 32 dépassaient déjà les $76 \mathrm{~m}^{13}$.

Figure 3 - Tours de plus de 100m construites à Londres entre 1960 et 2010 (et projection)

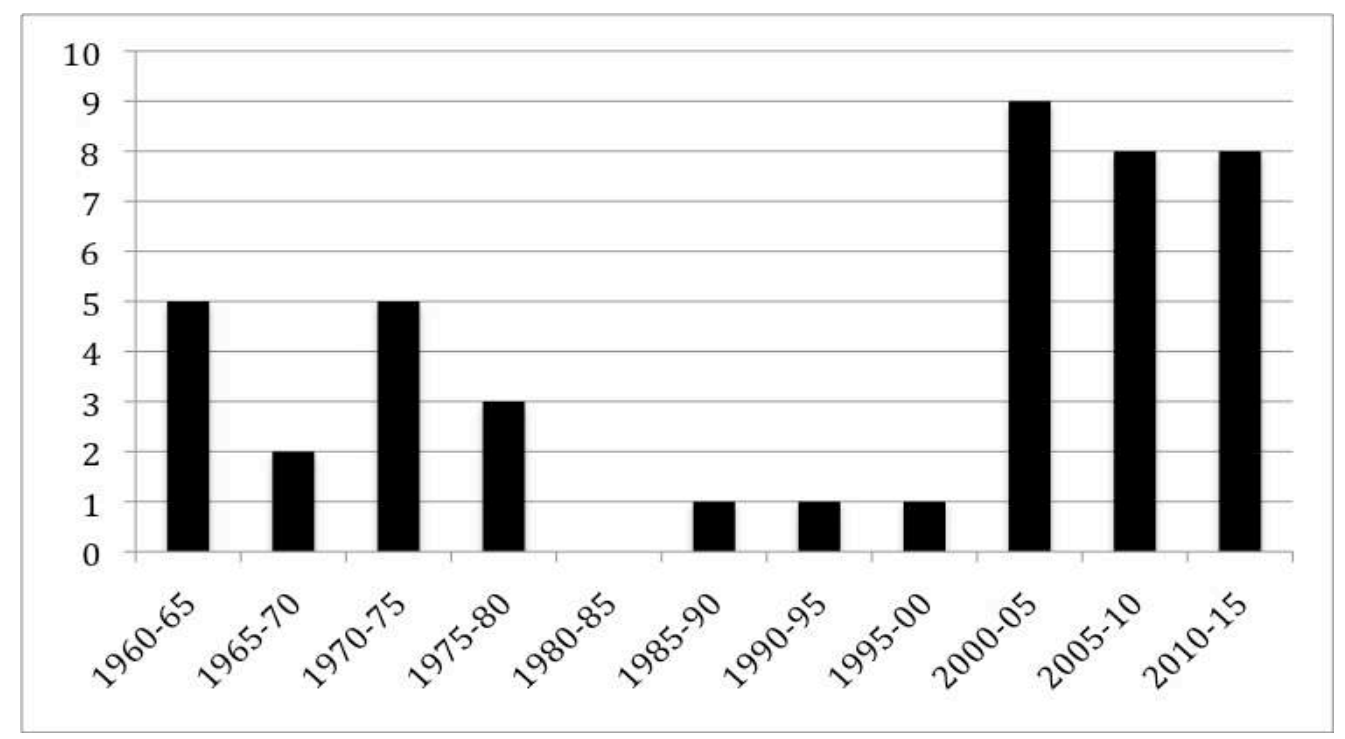

M. Appert, 2010

Conscients du besoin de reconstruction mais sensibles aussi à l'opinion publique qui était opposée aux tours, les urbanistes n'ont cessé de tergiverser jusqu'à la fin des années 1980, évaluant avec plus ou moins de sévérité les projets déposés. La période s'est donc caractérisée par une inconstance règlementaire qui a rendu floues les règles du jeu et qui a permis le percement de la canopée urbaine de façon plus accidentelle que planifiée ${ }^{14}$. Durant les deux décennies suivantes, les législations successives ont continué à refléter ce dilemme, mais cette fois dans un contexte de reprise de la croissance économique puis démographique. La pression pour la construction d'immeubles de bureaux s'est alors principalement manifestée dans la City où des groundscrapers ${ }^{15}$ ont remplacé des immeubles des années 1960 jugés obsolètes. Cette période a aussi vu l'émergence d'un quartier de tours planifiées sur les anciens docks de l'Isle of Dogs à Canary Wharf. Ce dernier s'est rapidement révélé un concurrent de la City, attirant plusieurs banques américaines à la suite de l'extension de la Jubilee Line.

Jusqu'à la création de la municipalité de Londres en 2000, la législation mise en œuvre pour réguler le skyline de Londres a traduit une tolérance vis-à-vis des tours. Assurer le développement de la ville et tout particulièrement celui de la City face aux autres métropoles ou même aux Docklands est resté un facteur déterminant pour dissuader les autorités de mettre en place un quasi moratoire sur les tours comme le faisait Paris.

Dès son élection en 2000, le nouveau maire de Londres, Ken Livingstone, se prononçait en faveur des tours y compris au moment où il consultait les acteurs de la ville pour l'élaboration du London

\footnotetext{
${ }^{13}$ T. Catchpole, London Skylines: A study of High Buildings and Views, London Research Centre, vol. 33, 1987.

${ }^{14} \mathrm{~S}$. Holmes, The history and effects of changes, past and present, to London's skyline. 2004, http://wwwusers.brookes.ac.uk/01231893, (consulté le 19 décembre 2011).

${ }^{15}$ Immeubles de grande hauteur (jusqu'à $100 \mathrm{~m}$ ) mais disposant de très grands plateaux leur conférant une masse plus horizontale que verticale.
} 
Plan. Le principe fondateur du plan stratégique qui sera publié en 2004 était d'abord de donner une orientation générale au développement futur de la métropole. En ce qui concerne la régulation des projets de tours, le document rendait compte de l'accent mis par la GLA sur les activités financières mais dans le même temps, il consacrait aussi la préservation de la mise en scène des monuments dans le skyline de la ville. Le London Plan s'est ainsi accompagné d'une directive spécifique à l'intégration des tours dans la ville : la London View Management Framework ${ }^{16}$ (LVMF). Avec cette directive, la GLA souhaitait clarifier les règles du jeu entre les parties intéressées (acteurs économiques, conservateurs du patrimoine, associations) et réduire ou annuler le recours à I'Etat Central via les enquêtes publiques. Ken Livingstone a alors qualifié cette législation de conciliation entre deux polarités en tension : le respect des vues des monuments historiques et le rayonnement économique international de Londres, ville globale. Concrètement, la LVMF inscrit statutairement la protection de quatre édifices emblématiques (cathédrale Saint Paul, Le Parlement, La Tour de Londres et Buckingham Palace), identifie la place située devant la nouvelle mairie comme lieu civique de contemplation, officialise des couloirs de vues protégées depuis des points stratégiques (collines, parcs et ponts) dans la ville tout en limitant leur largeur. Le document entend enfin minimiser les litiges en clarifiant les données de la négociation grâce à l'usage du géo-référencement des vues et des points de contemplation ${ }^{17}$ (figure 4). Il s'agit bien d'une négociation dans le processus d'urbanisme dans la mesure où il n'est pas formellement interdit de construire une tour dans un couloir de vue. Il est toutefois très difficile de la faire accepter à moins que le promoteur ne puisse convaincre l'arrondissement concerné, la municipalité et les acteurs de la protection du patrimoine que son projet est d'une très haute qualité architecturale. Ce que le promoteur Sellar Properties, soutenu par la GLA, le Borough de Southwark parvint à faire pour permettre la construction du Shard, tour de $310 \mathrm{~m}$, pourtant visible dans un couloir de vue protégée de Saint Paul depuis Parliament Hill ${ }^{18}$ (figure 2).

\section{Figure 4 - Dispositif de la vue protégée de la cathédrale Saint Paul depuis Primrose Hill}

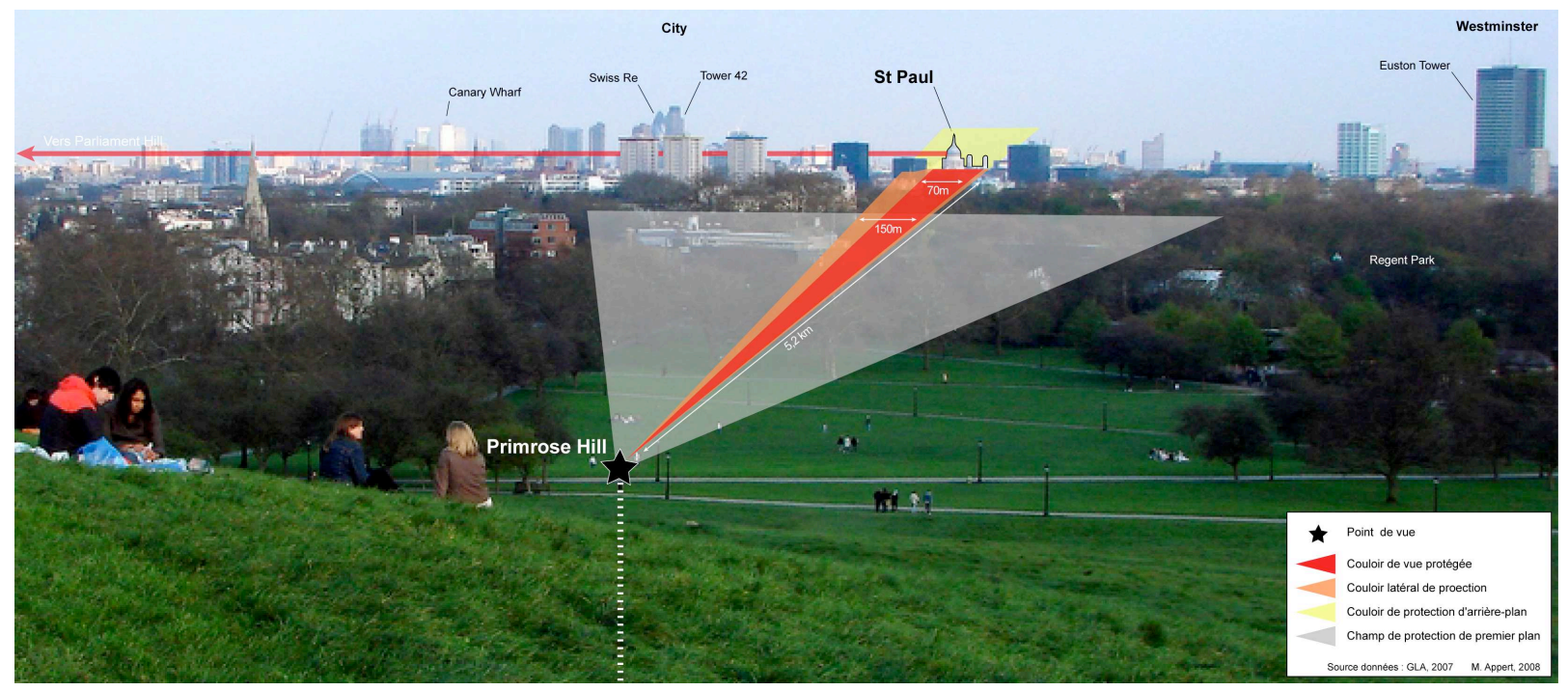

M. Appert, 2009

${ }^{16}$ G.L.A., London view management framework, 2007.

${ }^{17}$ A. Day, Urban visualization and public inquiries: the case of the Heron Tower, in Architectural Research Quarterly, vol. $6, n^{\circ} 4,2002$, p.363-372.

R. Tavernor, Visual and cultural sustainability: The impact of tall buildings on London, in Landscape and Urban Planning, vol. 83, 2007, p. 2-12.

${ }^{18}$ Appert M., "Ville globale versus ville patrimoniale ? Des tensions entre libéralisation du skyline de Londres et préservation des monuments et vues historiques ", in Revue Géographique de l’Est, vol. 48, n¹-2, 2008. 
L'exemple de Shard montre que la législation ne permet pas de clarifier totalement les règles du jeu. Si les couloirs de vues protégées tendent à dessiner des espaces du possible pour la construction de nouvelles tours, ils ne que concernent que les projets proposés dans Inner London et ce, depuis les années $1990^{19}$. Les localisation, hauteur, nature, fonction et acceptation des tours contemporaines relèvent aussi d'autres paramètres.

\section{Une géographie des tours de Londres}

Depuis l'approbation du permis de construire de la tour Heron dans la City en 2001 et la livraison du Gherkin en 2003, 67 projets de tours ${ }^{20}$ ont été déposés auprès des municipalités concernées, qu'il s'agisse de la City ou d'un grand nombre de boroughs à travers le Grand Londres. Fait nouveau dans la capitale britannique, la grande majorité des tours proposées sont à vocation résidentielle. Ce qui signifie que, au delà des tours de bureaux emblématiques, très médiatisées et affublées aujourd'hui de sobriquets - citons ici le Shard, le Cheesegrater, le Helter-Skelter - de nombreux projets restent relativement anonymes à l'échelle de la ville. Des tours résidentielles de hauteur plus mesurée, généralement comprise entre 80 et $150 \mathrm{~m}$, et d'un dessin plus conventionnel se multiplient depuis les années 2000 et constituent aujourd'hui l'essentiel de la cohorte d'immeubles de grande hauteur.

Cinquante deux des 67 projets de tours recensés (figure 5) dans le Grand Londres concernent le centre et le péricentre de la ville (Inner London). Apparaît donc ainsi une première caractéristique de la localisation du phénomène de verticalisation de Londres : une concentration dans l'espace le plus densément occupé. Ces tours ne sont pas réparties de façon homogène, car l'on constate d'abord une dissymétrie ouest-est (figure 6), avec la grande majorité des réalisations concentrées dans la partie centre-est de l'Inner London et notamment le centre de Londres, l'East End, jusqu'aux Royal Docks et dans une bien moindre mesure, la rive droite de la Tamise jusqu'à Woolwich.

Leur répartition spatiale fait apparaître plusieurs logiques. D'abord et sans surprise, une concentration des tours de bureaux dans les centres d'affaires de la City et Canary Wharf, deux des trois hubs financiers de la ville ${ }^{21}$. Un linéaire de tours résidentielles et hôtelières contestées se dessine ensuite le long des berges de la rive droite de la Tamise de Vauxhall à l'ouest, à Tower Bridge, à l'est. Vient ensuite une répartition plus ponctuelle de tours résidentielles autour de Vauxhall, Elephant and Castle ou bien Stratford dans Inner London, et Brent Cross, Barking, Ilford et Croydon, dans Outer London.

La hauteur de ces tours révèle par ailleurs un gradient de valeurs foncières centre-périphérie marqué, la City de Londres, Southwark et Canary Wharf concentrant l'intégralité des tours de plus de $200 \mathrm{~m}$ et présentant les coûts fonciers les plus élevés, alors que, à quelques exceptions près, aucune tour ne dépasse $110 \mathrm{~m}$ dans Outer London. Parmi ces exceptions, plusieurs projets de tours illustrent des tentatives d'élévation des valeurs du sol dans le contexte d'opérations de requalification urbaine menées conjointement par promoteurs et municipalités. Pour relativiser encore davantage ce schéma général, le West End de Londres, pourtant l'espace le plus onéreux de la capitale, échappe à la logique de verticalisation du fait de très fortes contraintes patrimoniales. Quant

\footnotetext{
${ }^{19}$ Mise en place des premiers couloirs de vues protégées en 1991. Voir DoE, Regional Planning Guidance 3, Annex $A$ (RPG3A): Supplementary Guidance for London on the Protection of Strategic Views, HMSO, 1991.

${ }^{20}$ En décembre 2009.

${ }^{21}$ Avec le West End et particulièrement les environs de Mayfair.
} 
au linéaire de tours le long de la Tamise, la multiplication des enquêtes publiques (Shard, Vauxhall, One Blackfriars et Doon Street), cristallise une grande partie des tensions entre conservateurs du patrimoine et bâtisseurs du Londres contemporain. Ces tours représentent à elles seules plus de la moitié des enquêtes publiques de la ville, toutes initiées par des opposants. En tentant de tirer profit de la vue sur les monuments de Londres et sur le fleuve, les promoteurs se sont heurtés à de vives oppositions émanant principalement de la Royal Park Agency ou même de la nouvelle municipalité de Londres, désormais dirigée par le conservateur Boris Johnson.

Figure 5 - Localisation des projets de tours de plus de $80 \mathrm{~m}$ à Londres en 2009

\begin{tabular}{|l|c|c|c|c|c|}
\hline $\begin{array}{l}\text { Secteurs } \\
\text { géographiques }\end{array}$ & $\begin{array}{l}\text { Nombre de } \\
\text { projets }\end{array}$ & $\begin{array}{l}\text { En } \\
\text { cours }\end{array}$ & Approuvés & Bureaux & $\begin{array}{l}\text { Enquêtes } \\
\text { publiques }\end{array}$ \\
\hline City de London & 7 & 3 & 2 & $89 \%$ & 2 \\
\hline Franges de la City & 12 & 1 & 4 & $14 \%$ & 0 \\
\hline Rive sud Tamise & 3 & 0 & 1 & $0 \%$ & 6 \\
\hline West End & 21 & 5 & 9 & $38 \%$ & 0 \\
\hline Canary Wharf & 15 & 1 & 6 & $7 \%$ & 1 \\
\hline Reste du Grand Londres & 5 & 0 & 2 & $20 \%$ & 0 \\
\hline \multicolumn{1}{|c|}{ Dont Croydon } & 67 & 13 & 29 & $30 \%$ & 10 \\
\hline Grand Londres & & & 5 & 0 \\
\hline
\end{tabular}

M. Appert, 2009

La géographie de la nature de l'occupation des tours londoniennes contemporaines tend à souligner le caractère concentrique de la répartition des fonctions métropolitaines mais indique aussi des bifurcations et révèle des potentiels conflictuels inégaux d'ouest en est.

La lecture simultanée de la carte et du tableau (figures 5 et 6 ) indique en effet que les projets de tours ont tendance à être calqués en grande partie sur la répartition des spécialisations fonctionnelles des différents espaces d'Inner London : services aux entreprises à forte valeur ajoutée dans la City et Canary Wharf, pour bénéficier d'économies $\mathrm{d}^{\prime}$ agglomération ${ }^{22}$, et tours résidentielles dans les quartiers déjà principalement caractérisés par cette fonction. La dissociation des fonctions, trait caractéristique de Londres dont l'hypercentre concentre plus de 1,2M d'emplois, semble donc se manifester aussi dans la verticalisation de la ville au point que les tours apparaissent comme des marqueurs du fonctionnement métropolitain. Toutefois, un changement notable dans la construction de tours depuis les années 1990 concerne la diversification des fonctions hébergées à Canary Wharf et la projection, aux marges de la City, de fonctions résidentielles et hôtelières ${ }^{23}$. Trente huit pour cent des projets dans le nouveau centre d'affaires des Docklands concernent ainsi des bureaux, ce qui contraste avec les $100 \%$ atteints durant la période 1991-2005. En ce sens, Canary Wharf tendrait à devenir une ville dans la ville, incorporant des fonctions ancillaires jusque là rejetées.

\footnotetext{
${ }^{22}$ M. Appert, Londres, métropole globale ? in Géocarrefour, vol. 83, n², 2008, p.113-8.

N. Buck et al., Working capital. Life and labour in contemporary London, Routledge, 2002, 408 p.

${ }^{23}$ Voir l'article de Martine Drozdz, dans ce même numéro.
} 


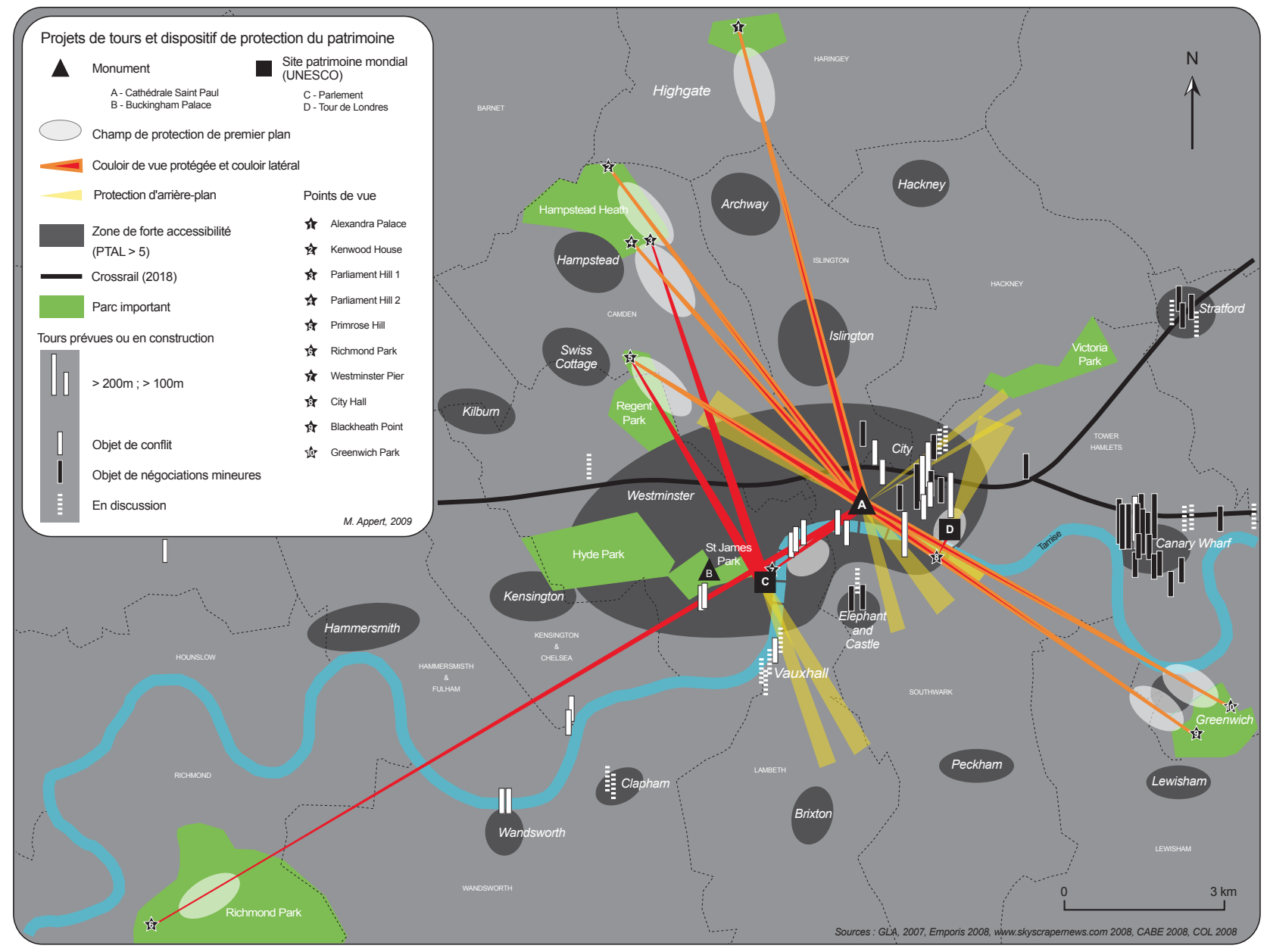

M. Appert, 2009

La City connaît, de son côté, un processus inverse car les quelques sites identifiés pour des tours (Eastern Cluster ${ }^{24}$ ) font l'objet de tant de convoitises que les promoteurs, qui cherchent avant tout à rentabiliser leurs investissements, arbitrent en faveur de complexes de bureaux, plus lucratifs. En lien avec la Corporation of London ${ }^{25}$, les arrondissement limitrophes, dans lesquels se concentrent pauvreté et précarité, ont plus souvent accepté que les promoteurs construisent des logements et hôtels pour la $\mathrm{City}^{26}$, en échange de contributions financières (Planning gains) à destination notamment des services publics locaux. L'East End de Londres est ainsi (re)devenu un terreau plus fertile aux tours et ce malgré les résistances suscitées par plusieurs projets proposés depuis 2007. Nous pouvons avancer plusieurs explications. D'abord, l'East End se caractérise par une plus faible proportion de constructions antérieures à 1960 que l'on doit notamment à la concentration et à l'intensité des bombardements du port de Londres pendant la seconde guerre mondiale. Corrélativement, l'existence de tours de logements sociaux a contribué à y modifier davantage l'élévation des formes urbaines et, si l'on y ajoute une concentration de population en transition souvent issue de l'immigration, il est vraisemblable que cette partie de la ville présente moins de raisons de résister aux tours. Inversement, le développement important de la City, l'émergence de

\footnotetext{
${ }^{24}$ Corporation of London, Saint Paul's and Monument views, Supplementary Planning Guidance, London, 2007,26 p.

${ }^{25}$ Municipalité de la City.

${ }^{26}$ M. Appert, M. Drozdz, La géopolitique locale-globale aux marges de la City de Londres : conflits autour des projets de renouvellement urbain de Bishopsgate, in Hérodote, ${ }^{\circ} 137,2010$, p. 119-134.
} 
Canary Wharf comme nouveau centre d'affaires et la désignation de Stratford pour accueillir les JO 2012 constituent des vecteurs de pression foncière majeurs qui diffusent en leurs marges les velléités de verticalisation.

La géographie des tours à Londres serait-elle alors uniquement guidée par les lois du marché foncier et la recherche d'économies d'agglomération ? Après 6 ans de planification stratégique depuis la publication du London Plan, les directives d'aménagement à l'échelle du Grand Londres ou des boroughs ont-elles eu un impact sur la localisation, la nature et la hauteur des tours ?

La législation sur la protection des vues des monuments de Londres est probablement la plus déterminante pour la localisation des tours en définissant des possibles. La présence ou au contraire l'absence d'un couloir de vue en un lieu est en effet une des conditions préalables à l'élaboration d'un projet de renouvellement urbain vertical. Même si Shard a pu être construite dans un couloir de protection d'arrière-plan de St Paul's depuis Parliament Hill, aucun autre promoteur n'a depuis tenté de braver la législation (figure 6). II faut dire que les sites pouvant accueillir des tours se sont multipliés dans la deuxième partie des années 2000. Les municipalités, sous l'autorité de la GLA, ont dû en effet identifier en leur sein des sites susceptibles de les recevoir. La Corporation of London a ainsi délimité à l'est de son territoire un espace de concentration de tours de très grande hauteur (Eastern Cluster).

Moins directement lié aux tours, le London Plan ${ }^{27}$ (2004) n'en est pas moins structurant dans la mesure où, dans une perspective de long terme, il devient un environnement urbanistique dans lequel les boroughs déterminent les permis de construire. Ce plan stratégique rompt avec les politiques de containment urbain menées jusque dans les années 1990 pour embrasser la croissance économique et l'essor démographique retrouvés de la métropole à la fin du XXème siècle. Pour cela, il s'inspire d'une part des préceptes de la ville compacte et de l'autre il fait sien, notamment du fait de la personnalité de Ken Livingstone, du rôle global de Londres. Sont donc favorisées : la densification urbaine en lien avec la localisation des pics d'accessibilité en transports collectifs, la requalification des friches urbaines et la verticalisation du centre-vitrine et des abords des hubs de transport ${ }^{28}$. C'est dans cette logique qu'ont été pensées les transformations d'Elephant and Castle, de Vauxhall ou du Bishopsgate Goodsyard. II n'est donc pas étonnant que les tours soient devenues une solution privilégiée pour les opérations de " régénération " urbaine. L'orientation donnée par le London Plan a incité les promoteurs à privilégier cette forme urbaine alors même qu'il n'est pas avéré que les tours permettent de maximiser l'usage du sol ${ }^{29}$. La tour est devenu symbole de "régénération ", tel un flambeau qui marque le paysage en rendant lisibles les transformations urbaines. L'East End de Londres est symptomatique de ce phénomène, car parmi les rares constructions anciennes et les immeubles construits après la seconde guerre mondiale, se dressent aujourd'hui des dizaines de tours résidentielles, telles une signalétique de la transformation physique mais aussi sociale et symbolique de l'espace. Si les dynamiques socio-économiques ne peuvent expliquer à elles seules la géographie des tours londoniennes, elles restent déterminantes dans la mesure où l'urbanisme local prend appui sur le secteur privé, à la fois comme source de financement, mais aussi comme inspirateur du sens donné à la planification stratégique contenue dans le London Plan.

\footnotetext{
${ }^{27}$ G.L.A., The London plan, 2004, 420 p.

${ }^{28} \mathrm{M}$. Appert, Métropolisation, mobilités quotidiennes et forme urbaine : le cas de Londres, in Géocarrefour, vol. 79, $\mathrm{n}^{\circ} 2,2004$, p. 109-118.

${ }^{29}$ House of Commons, Tall building report, 2002.
} 


\section{Conclusion}

Le retour des tours à Londres, particulièrement spectaculaire depuis l'arrivée de Ken Livingstone aux affaires en 2000, est une des incarnations des mutations socio-économiques et politiques de Londres au tournant du XXlème siècle. Les projets de tours font apparaître une reproduction des spécialisations fonctionnelles des espaces de la métropole, soulignant et renforçant la concentration des activités financières dans l'hyper-centre. Ils confirment aussi, à l'exception de la diversification de Canary Wharf, la séparation des fonctions dans la ville ainsi que la régénération de l'East End, en proie à une transformation paysagère et sociale importante. Les tours rendent finalement lisible le London Plan en inscrivant dans le paysage l'intensification urbaine, en traduisant une gouvernance fondée sur les partenariats public-privé pour la requalification urbaine et en révélant la rhétorique de la ville globale, si chère à Ken Livingstone, mais finalement aussi, à son successeur.

\section{Bibliographie}

Appert M., "Métropolisation, mobilités quotidiennes et forme urbaine : le cas de Londres ", in Géocarrefour, vol.79, n², 2004, p. 109-118.

Appert M., "Londres, métropole globale ? ", in Géocarrefour, vol.83, n², 2008, p. 113-118.

Appert M., "Ville globale versus ville patrimoniale ? Des tensions entre libéralisation du skyline de Londres et préservation des monuments et vues historiques ", in Revue Géographique de l'Est, vol. 48, n¹-2, 2008. http://rge.revues.org/index1154.htm, Consulté le 12 janvier 2011.

Appert M. et Drozdz M., "Conflits d'aménagement aux marges nord-est de la City de Londres » in Hérodote, $n^{\circ} 137,2010$, p. 119-134.

Buck N. et al., Working capital. Life and labour in contemporary London, Routledge, London, 2002, $408 \mathrm{p}$.

Catchpole T., London Skylines: A study of High Buildings and Views, Londres, London Research Centre, vol.33, 1987.

Corporation of London, Saint Paul's and Monument views, Supplementary Planning Guidance, London, 2007, 26 p.

Day A. 2002, "Urban visualization and public inquiries: the case of the Heron Tower", in Architectural Research Quarterly, vol.6, n4, 2002, p. 363-372.

Dennis R., "'Babylonian Flats' in Victorian and Edwardian London", in The London Journal , vol.33, $n^{\circ} 3,2008$, p. 233-247.

DoE, Regional Planning Guidance 3, Annex A (RPG3A): Supplementary Guidance for London on the Protection of Strategic Views, London, 1991.

G.L.A., The London Plan, Londres, 2004, 420 p.

G.L.A., London view management framework, Londres, 2007, 388 p.

Graham S., Marvin S., Splintering Urbanism: Networked Infrastructures, Technological Mobilites and the Urban Condition, Routledge, London, 2001.

Holmes S., The history and effects of changes, past and present, to London's skyline. 2004, http://wwwusers.brookes.ac.uk/01231893, consulté le 19 décembre 2011.

House of Commons, Tall building report, London, 2002. 
Kostoff S., The City Shaped: Urban Patterns and Meanings Through History, Thames \& Hudson, London, 2001.

McNeill D., "Skyscraper geography", in Progress in Human Geography, vol.29, n¹, 2005, p. 41-55.

Monnet J., "La symbolique des lieux : pour une géographie des relations entre espace, pouvoir et identité ", in Cybergeo, Politique, Culture, Représentations, article 56, mis en ligne le 07 avril 1998, modifié le 03 mai 2007. URL: http://www.cybergeo.eu/index5316.html. Consulté le 16 janvier 2011.

Simon R.D., "Skyscrapers and the New London Skyline: 1945-1991", The Electronic Journal of Architecture, vol.5, $\mathrm{n}^{\circ} 2$, 1996. http://corbu2.caed.kent.edu/architronic/v5n2/v5n2.06a.html, consulté le 05 janvier 2011.

Tavernor R., "Visual and cultural sustainability: The impact of tall buildings on London", in Landscape and Urban Planning, vol. 83, 2007, p. 2-12.

Urban Task Force, Toward an urban Renaissance, London, 1999, 20 p.

Wright H., London high. A guide to past, present, and future of London's skyscrapers, Frances Lincoln, London, 2006. 